\title{
Wavelet Statistical Texture Features with Orthogonal Operators Tumour Classification in Magnetic Resonance Imaging Brain
}

\author{
${ }^{1}$ R. Meenakshi and ${ }^{2}$ P. Anandhakumar \\ ${ }^{1}$ Department of I.T., Saveetha Engineering College, Chennai, Tamilnadu, India \\ ${ }^{2}$ Department of I.T, M.I.T Campus, Chennai, Tamilnadu, India
}

Received 2013-08-06, Revised 2013-08-19; Accepted 2013-08-23

\begin{abstract}
Tumors medically also called neoplasms are an abnormal mass of tissue resulting from uncontrolled proliferation or division of cells occurring in the human body. If such growth is located in the brain then it is called as brain tumor. Identification of such tumors is a major challenge in the field of medical science. Early identification of tumors prove to be critical as serious consequences can be averted. Its threat level depends on a combination of various factors like the type of tumor, its location, its size and its developmental stage. Tumor can occur in any part of the body. Magnetic Resonance Imaging (MRI) technique is mainly used for analyzing the brain, as the images produced are of high precision and applicability. The main objective of this study is to classify the brain MRI dataset for the existence or non existence of tumors. The proposed method uses Two Dimensional Discrete Wavelet Transform (2D-DWT) for pre-processing and further classification with orthogonal operators and SVM. The usage of 2D-DWT for pre-processing improves the classification accuracy by $2 \%$ when compared to the existing classification techniques.
\end{abstract}

Keywords: MRI, Discrete Wavelet Transform, Support Vector Machine (SVM), Orthogonal Polynomial Operator, Orthonormal Operator

\section{INTRODUCTION}

A tumor is a mass of tissue that grows out of control of the normal forces that regulates growth (Kotsas, 2005). Brain tumor is one of the major causes for the increase in mortality among children and adults (Jaya et al., 2009). Early detection and proper treatment based on accurate diagnosis are important steps to improve the survival rate among humans. For detection of brain tumor, MRI has become one of the most common tool.

MRI is a non invasive medical imaging technology that has proven to be an effective tool in the study of the human brain (Bhandarkar and Nammalwar, 2001). The information that MRI provides has become important in the field of medical research. The knowledge gained from this data has become a critical component in diagnosis and treatment planning (Archibald et al., 2003) of brain tumors.

Corresponding Author: R. Meenakshi, Department of I.T., Saveetha Engineering College, Chennai, Tamilnadu, India
This technology has greatly improved the sensitivity and specificity of diagnostic imaging. In the last decades, many methods have been proposed for segmentation of tumor in brain MRI. Some of the methods use neural networks (Sammouda et al., 1996) and some of them use finite Gaussian mixture model (Moon et al., 2002). Knowledge-based methods (Clark et al., 1998), atlas based method, active contour model (Cuadra et al., 2004), level set methods (Lefohn et al., 2003) and outlier detection (Prastawa et al., 2004) have also been used for segmentation of tumor in brain MRI.

\subsection{Related Works}

Morris et al. (2006) has used some pre-processing techniques on brain MRI for classification of glioma. The techniques that he used were noise reduction for 
inter slice intensity variation reduction, registration was used to linearly register MRI to a standard co ordinate system, intensity registration was used to reduce the intensity among different MRI, tissue segmentation was used to differentiate between grey matter, white matter and Cerebro Spinal Fluid CSF (Morris et al., 2006). Yang and Huang (2006) has used modified curvature diffusion equation as pre-processing technique to enhance and preserve edges in brain MRI (George and Karnan, 2012).

George and Karnan (2012) in his study, has done the removal of artifacts and modified tracking algorithm as a pre-processing step. The removal of artifacts was used to remove high intensity values from the image and the modified tracking algorithm was used to remove the un wanted portion of the image (George and Karnan, 2012). Noise reduction is done as pre-processing step in image classification according to (Sebe and Michael, 2000). The author has analysed the use of gabor and QMF filters for noise reduction purpose (Sebe and Michael 2000). Patil and Udupi (2012) in his study has emphasized the need for pre-processing. The author says the process is needed in order to remove artifacts which hinder further processing of MRI. Pre-processing of images is needed for processing them using ComputerAided Diagnosis (CAD). The author has used median filtering and square shaped structuring element as a preprocessing step in his study (Patil and Udupi, 2012).

Jaya et al. (2009) have used removal of artifacts as a pre-processing step. The author has set a threshold value of 255 for artifacts removal and a threshold value of 200 for the removal of unwanted portion of the image (Jaya et al., 2009). Ratan et al. (2009) in his study has used a different type of pre-processing for reducing the processing amount of data. He at a time processes a whole of 128 images into 3 clips (Ratan et al., 2009). Bauer et al. (2013) survey paper, the author has analysed many papers that had dealt with image preprocessing technique. The author says that even though many techniques remove noise, they introduce a negative effect which affect the segmentation of the image (Wang and Min, 2009).

A different method called a Multivariate Iterative Region Growing Using Semantics (MIRGS) was used to detect the tumor. In this Region level K-Means (RKM) is used. This is based on initialization method that consistently provided accurate initial conditions at low computational cost. Some of the experiments showed its superiority to RKM relative to two commonly used initialization methods (Qin and Clausi, 2010).
Another methodology for automatic segmentation of heterogeneous image data reduced the gap between bottom-up affinities based segmentation methods and top-down generative model. These approaches, aimed at detecting the tumor, ran faster giving improved results when compared to other methods. Results produced by quantitative analysis indicated the benefit of incorporating model affinities into the segmentation process for the difficult case of brain tumor (Corso et al., 2008).

Another novel approach, called region-based method for image segmentation, used regions which deal with intensity inhomogeneities in the segmentation. This methodology was validated on synthetic images and real images of various modalities and desirable performance was obtained in the presence of intensity inhomogeneities. Performance evaluation showed that this method was robust to initialization and made for faster and accurate detection (Wang and Min, 2009).

The algorithm of BPN has been used in various fields, right from computer network security to sonar image classification. BPN and SVM are used for classification of MRI in the present work.

\section{MATERIALS AND METHODS}

In this proposed method classification of brain MRI is done using the combination of 2D-DWT for preprocessing and classification using SVM. Twodimensional discrete wavelet decomposition is performed on the tumour image to remove the noise and to make the image coarser and also to reduce the size of the original image. Classification of tumour from non tumour is done by a Support Vector Machine (SVM).

This proposed work presents a method of preprocessing the image with wavelet transform. On this image orthogonal operators are applied. The resultant is a feature space which is grouped into two. The result of this group for all the images is given to SVM as input. The performance of SVM classifier is measured in classifying tumour from non tumour images. The combination of wavelet transform with orthogonal operator has been proposed in this work using SVM classifier enabling proper classification thereby reducing the complexity involved. The developed classification system is expected to provide a valuable and accurate classification process for the physicians. This proposed method, gives the highest classification accuracy of $99 \%$ when compared with other conventional texture analysis methods. 
System architecture

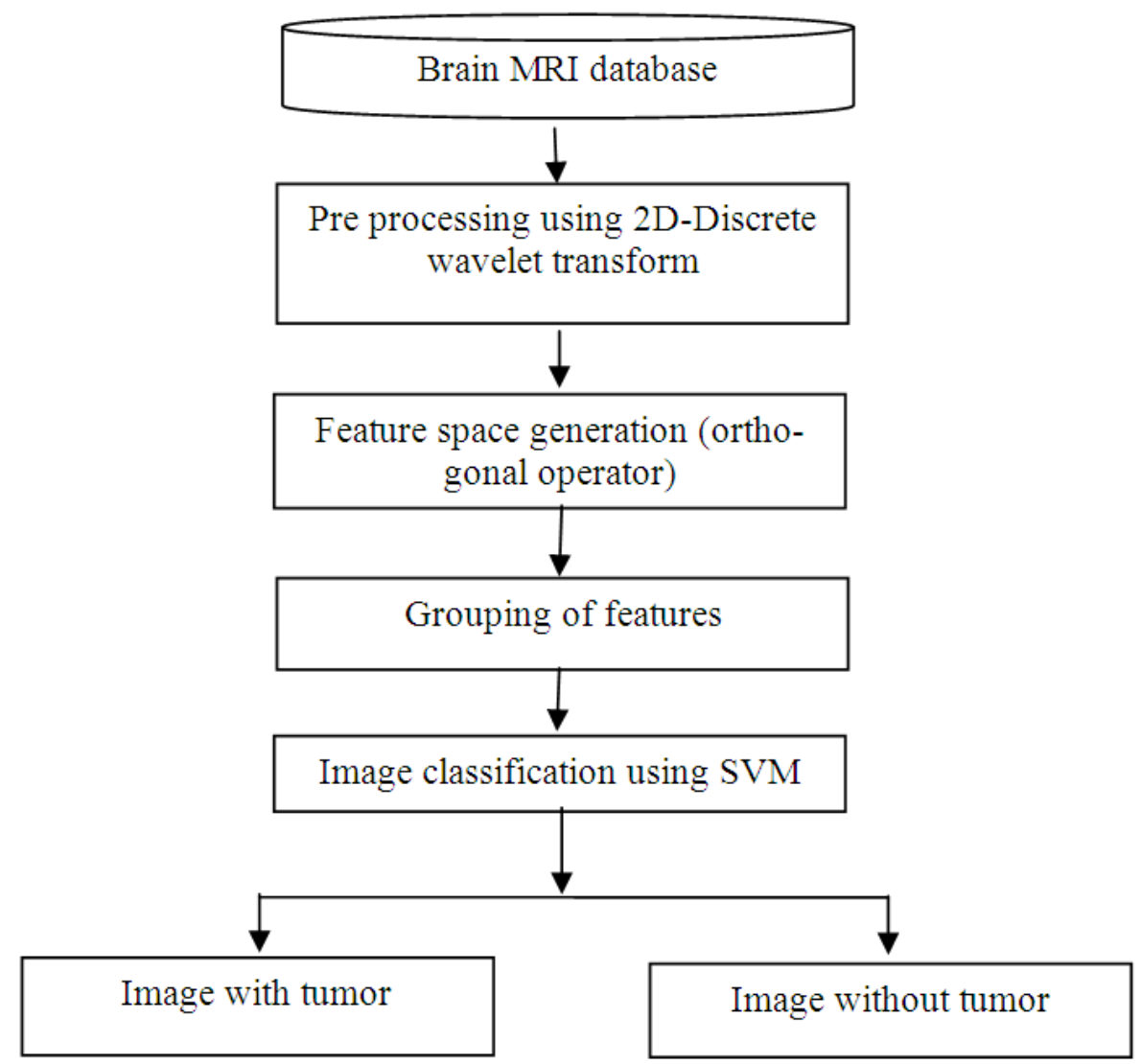

Fig. 1. System architecture

The system architecture of the proposed work is shown in Fig. 1. This figure shows the steps that the proposed work follows while classifying the tumor MRI from the database. From the database of brain MRI's each image is considered for processing. The database consists of a total of 172 images. All the images are taken for training and testing. In these 172 images, 97 are tumor images and 75 are non-tumor images. The size of the images is either $512 \times 512$ or $256 \times 256$.

The performance of the proposed technique in tumor identification is evaluated by using the positive and negative cases. This means if a brain MRI is one with tumor then it must be identified as a tumor image i.e., TRUE(T) and POSITIVE(P). The other combinations in this include:

- TRUE NEGATIVE (TN)-In this case the given tumor image is not identified as one
- FALSE POSITIVE (FP)-In this case the given nontumor image is identified as one

- FALSE NEGATIVE (FN)-In this case the given non-tumor image is not identified as one

Using these cases the sensitivity, specificity and accuracy are defined as follows Equation 1-4:

Sensitivity $=\mathrm{TP}+\mathrm{FN}$

Specificity $=\mathrm{TN} /$ Total no. of tested images

Accuracy $=$ Sensitivity $/$ Total no. of tested images

Total percentage of accuracy $=$ Accuracy $* 100$

At the outset the image is pre-processed using a two Dimensional Discrete Wavelet Transform (2D-DWT). 
This is done in order to get a lossless compressed image, denoised image (Chang et al., 2000) as well as to make the image coarse. This coarse image gives us a clear image as that of the original image and also the whole size of the images is reduced to $75 \times 75$ without affecting the intensity values. This helps in the faster calculation of feature space. The next step is to apply the orthogonal operator on this image to obtain a feature space. This feature space is grouped into two using histograms. This result is given to SVM for classification.

\section{RESULTS AND DISCUSSION}

The evaluation metrics used as TP, FN, TN and FP for evaluating the percentage of accuracy in identifying the tumor in the given image. Here TP means True Positive, FN means False Negative, TN True Negative, FP means False Positive. The combination of 2D-DWT and classification is used to identify the tumor in a MRI of the brain. Orthogonal operators of sizes 3,4 and 5 are used and all the sets of these sizes are applied on the image for further process and evaluation. The grouping is done using the built-in hist operator which groups the whole feature space based on the values of the feature space. Therefore instead of finding the features from the feature space the values are divided into two thereby reducing the length of the vector formed just to two. This result is given to SVM for classification of tumor in the image.

Table 1 shows the values of the evaluation parameters TP, FN, TN and FP. The total images considered are 172 out of which 97 are tumor images and 75 are non-tumor images which are identified correctly.

Table 1. Sensitivity factor for various sizes of orthogonal operators combined with SVM

\begin{tabular}{lrrr}
\hline Operator size & $3 \times 3$ & \multicolumn{1}{c}{$4 \times 4$} & $5 \times 5$ \\
\hline TP & 87.00 & 94.00 & 97 \\
FN & 53.00 & 720.00 & 75 \\
TN & 0.00 & 0.00 & 0 \\
FP & 32.00 & 6.00 & 0 \\
Percent of accuracy & 81.39 & 96.51 & 100 \\
\hline
\end{tabular}

Table 2. Sensitivity factor for various sizes of orthonormal operators combined with SVM

\begin{tabular}{lrrr}
\hline Operator size & $3 \times 3$ & $4 \times 4$ & $5 \times 5$ \\
\hline TP & 82.00 & 93.00 & 96.00 \\
FN & 53.00 & 69.00 & 75.00 \\
TN & 0.00 & 0.00 & 0.00 \\
FP & 37.00 & 10.00 & 1.00 \\
Percent of accuracy & 78.48 & 94.18 & 99.41 \\
\hline
\end{tabular}

This correct identification is done for the orthogonal operator size of 3,4 and also 5. Table 2 shows the value details when orthonormal operators are used for the same purpose as that of orthogonal operator. Both the operators give the same percentage of accuracy. Figure 2 shows the evaluation metrics for sizes 3,4 and 5 showing them for both SVM and BPN classifiers. SVM gives $100 \%$ accuracy for the orthogonal operator size $5 \times 5$ whereas BPN gives only $52.9 \%$ for this size.

\subsection{Limitations \\ 3.1.1. Comparing with Existing Work}

The present research work is limited images of brain MRI. It can extend to other MRI images of liver or breast. It could also be extended to colour images if needed or even videos.

Table 3. Comparative study of proposed work with existing techniques

Co-occurrence texture features using

bidirectional associative memory

Optimal dominant grey-level run length

texture features using SVM classifier

combined co-occurrence, grey level and new

edge features using SVM classifier

$93.75 \%$

$95.80 \%$

Wavelet co-occurrence texture features to

classify the normal, benign and malignant

tumour images using SVM-based classifier

Wavelet co-occurrence texture

features using a PNN classifier

Wavelet-based dominant run length and co-occurrence texture features classified to classify the normal, benign and malignant tumour images using SVM-based classifier Proposed combination of 2D-DWT for pre processing, orthogonal operator and classification using SVM to

classify normal and tumor images.

$96.25 \%$

$96.29 \%$

$97.00 \%$

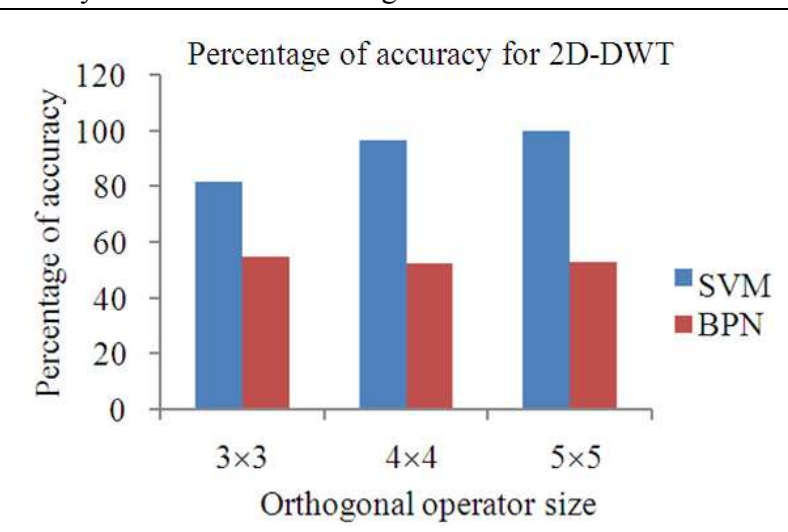

Fig. 2. The percentage of accuracy for various operator sizes and classifiers 


\section{CONCLUSION}

Tumor classification, even though is a tough job, has been made easy using the proposed work of use of 2DDWT and orthogonal operators since it is automated and DWT reduces the size of the image without any loss and the feature space generated is very small to the size of $75 \times 75$ when compared to $256 \times 256$ Table 3 .

The orthogonal polynomial and orthonormal operators used here are the most commonly used masking operators used to generate the feature space of the given image. In these operators for each size all the operators i.e., for example for size 3 all the sets from set 2 to set 8 are considered. Each of these are used with a brain MRI to get a feature space. The feature space is reduced using histograms and the result is classified using SVM. For comparison BPN was also tried on. Experiments were done without using pre processing for the operator sizes 3,4 and 5 and considering all its sets. The accuracy was only $72 \%$.

The proposed technique is effective since it has produced a detection accuracy of $100 \%$ for the operator size $5 \times 5$ as a result of applying a pre-processing of DWT on the image and combining it with SVM classification. This research work can be further extended by using the optimal operator set. This can also be extended for different sizes of orthogonal polynomial operators.

\section{REFERENCES}

Archibald, R., K. Chenb, A. Gelbc and R. Renaut, 2003. Improving tissue segmentation of human brain MRI through preprocessing by the Gegenbauer reconstruction method. Neuro. Image, 20: 489-502. PMID: 14527609

Bauer, S., R. Wiest, L. Nolte and M. Reyes, 2013. A survey of MRI-based medical image analysis for brain tumor studies. Phys. Med. Biol., 58: 97-129. PMID: 23743802

Bhandarkar, S.M. and P. Nammalwar, 2001. Segmentation of multispectral MR images using a hierarchical self-organizing map. Proceedings of the 14th IEEE Symposium on Computer-Based Medical System, (MS' 01), IEEE Xplore Press, Bethesda, MD, $\quad$ pp: 294-299. DOI: 10.1109/CBMS.2001.941735

Chang, S., G.B. Yu and M. Vetterli, 2000. Spatially adaptive wavelet thresholding with context modeling for image denoising. IEEE Trans. Image Proc., 9: 1522-1531. DOI: 10.1109/83.862630
Clark, M., L. Hall, D. Goldgof, R. Velthuizen and F. Murtagh et al., 1998. Automatic tumor segmentation using knowledge-based techniques. IEEE Trans. Med. Imag., 17: 187-201. DOI: 10.1109/42.700731

Corso, J.J., E. Sharon, S. Dube, S. El-Saden and U. Sinha et al., 2008. Efficient multilevel brain tumor segmentation with integrated Bayesian model classification. IEEE Trans. Med. Imag., 27: 629640. DOI: 10.1109/TMI.2007.912817

Cuadra, M., C. Pollo, A. Bardera, O. Cuisenaire, J.G. Villemure and J.P. Thiran, 2004. Atlas-based segmentation of pathological MR brain images using a model of lesion growth. IEEE Trans. Med. Imag., 23: 1301-1314. PMID: 15493697

George, B.E. and M. Karnan, 2012. MRI brain image enhancement using filtering techniques. Int. J. Comput. Sci. Eng. Technol., 3: 399-403.

Jaya, J., K. Thanushkodi and M. Karnan, 2009. Tracking algorithm for de-noising of MR brain images. Int. J. Comput. Sci. Net. Security, 9: 262-267.

Kotsas, P., 2005. Non-rigid registration of medical images using an automated method. World Acad. Sci. Eng. Technol., 1: 199-201.

Lefohn, A.E., J.E. Cates and R.T. Whitaker, 2003. Interactive, GPU-based level sets for 3D segmentation. Proceedings of Medical Image Computing and Computer-Assisted Intervention, Nov. 15-18, Canada, pp: 564-572. DOI: 10.1007/978-3-540-39899-8_70

Moon, N., E. Bullitt, K.V. Leemput and G. Gerig, 2002. Automatic brain and tumor segmentation. Proceedings of 5th International Conference on Medical Image Computing and Computer-Assisted Intervention, Sept. 25-28, Tokyo, Japan, pp: 372379. DOI: 10.1007/3-540-45786-0_46

Morris, M., R. Greiner, J. Sander, A. Murtha and M. Schmidt, 2006. Learning a classification-based glioma growth model using MRI data. J. Comput., 1: 21-31. DOI: $10.4304 /$ jcp.1.7.21-31

Patil, S. and V.R. Udupi, 2012. Preprocessing to be considered for $\mathrm{MR}$ and $\mathrm{CT}$ images containing tumors. J. Electr. Electron. Eng., 1: 54-57.

Prastawa, M., E. Bullitt, S. Ho and G. Gerig, 2004. Robust estimation for brain tumor segmentation. University of North Carolina.

Qin, A.K. and D.A. Clausi, 2010. Multivariate image segmentation using semantic region growing with adaptive edge penalty. IEEE Trans. Image Proc., 19: 2157-2170. DOI: 10.1109/TIP.2010.2045708

Ratan, R., S. Sharma and S. Sharma, 2009. Multiparameter segmentation and quantization of brain tumor from MRI images. Int. J. Sci. Technol., 2: 11-15. 
Sammouda, R., N. Niki and H. Nishitani, 1996. A comparison of Hopfield neural network and Boltzmann machine in segmenting MR images of the brain. IEEE Trans. Nucl. Sci., 43: 3361-3369. DOI: $10.1109 / 23.552753$

Sebe, N. and S.L. Michael, 2000. Wavelet based texture classification. Proceedings of the 15th International Conference on Pattern Recognition, (PR' 00), pp: 3959-3962.
Wang, X.F. and H. Min, 2009. A level set based segmentation method for images with intensity inhomogeneity. Emerg. Intell. Comput. Technol. Appli., 5755: 670-679. DOI: 10.1007/978-3-64204020-7_72

Yang, Y. and S. Huang, 2006. Novel statistical approach for segmentation of brain magnetic resonance imaging using an improved expectation maximization algorithm. Optica Applic., 36: 125-136. 